\section{Chilled storage of zebrafish embryos: Development of an efficient protocol}

\author{
Maria Sampieri, Erika Molica Colella, \\ Silvia Dotti
}

Istituto Zooprofilattico Sperimentale della Lombardia e dell'Emilia Romagna - IZSLER, Centro di Referenza Nazionale per i Metodi Alternativi, Benessere e Cura degli Animali da Laboratorio, Brescia, Italy

\begin{abstract}
Making the zebrafish embryo available to be able to share it between different laboratories groups could pave the way for this animal model with the aim of disseminating and promoting the use and application of zebrafish in scientific research.
\end{abstract}

\section{Introduction}

The development of new methods aimed at preserving embryos is currently an unresolved problem: nowadays the procedures available for some aquatic species are low-speed freezing and vitrification with highly concentrated solutions of cryoprotectants. Indeed, there isn't one single and functional protocol for the conservation and cooling storage of zebrafish embryos focused at preserving their structure, functionality and morphology. Therefore, the identification of a practical procedure, which can be used in different laboratories and which, above all, is efficient, could broaden new horizons in the conoscitive and shared context between laboratories. Also, the availability of preserved embryos would be an added value and could have an important impact not only on the preservation of genetically modified lines, but also on trade. As a result, the goal was to identify the ideal temperature to arrest embryo's development, point out the best storage solution to use and the maximum incubation period in order to determine a procedure to guarantee the optimal conservation of zebrafish embryos.

The protocol that we have tried to build, step by step, takes its cue primarily from the work of Desai and colleagues, ${ }^{1}$ but other studies have also guided us in practical operations and decisions. In fact, several variables come into play in the identification of a cooling protocol for zebrafish embryos.

\section{Materials and Methods}

Firstly, to set our protocol it was decided to start basing on what is reported in literature and then to modify and adjust it basing on the results obtained from the tests previously carried out.

For this reason, the values reported below are the ones we have selected after having consulted the bibliography:2,3 i) Embryo's developmental stage: 24 hours post-fertilization (hpf); ii) Storage solutions: Dimethyl sulfoxide (DMSO) and Methanol (MeOH); iii) Concentration range of storage solutions: from $1 \mathrm{M}$ in the first test to $0.025 \mathrm{M}$ in the last one; iv) Negative control: Embryo Medium (EM); v) Sucrose: yes, used from the second experiment; vi) Exposure time: 24 hours of incubation; viability checked at 24 hours post treatment (hpt) and 96 hpt; vii) Exposure temperature: $+4^{\circ} \mathrm{C}$; viii) Post-cooling survival, deformity rates and possible structural damages registered.

As regards the execution of the method, the fish were placed in the mating tank at a ratio males:females of $2: 1$. The next day, in the early morning, the eggs were collected and transferred to Petri dishes at a temperature of $28.5^{\circ} \mathrm{C}$.

The following day, at 24 hpf the eggs were examined under a microscope to select and count only the viable ones. Then, the
Correspondence: Maria Sampieri, Istituto Zooprofilattico Sperimentale della Lombardia e dell'Emilia Romagna - IZSLER, Centro di Referenza Nazionale per i Metodi Alternativi, Benessere e Cura degli Animali da Laboratorio, Brescia, Italy.

E-mail: maria.sampieri@izsler.it

Key words: Zebrafish; embryo; chilled storage.

Acknowledgments: This study was financially supported by the Italian Ministry of Health.

Disclosures: The authors declare no conflict of interest.

Conference presentation: This paper was presented at the Third Centro 3R Annual Meeting - L'era delle 3R: modelli in silico, in vitro e in vivo per promuovere la ricerca traslazionale 30 September - 1 October 2021, Evento online organizzato dal Politecnico di Torino.

Received for publication: 9 July 2021.

Accepted for publication: 7 September 2021.

This work is licensed under a Creative Commons Attribution NonCommercial 4.0 License (CC BY-NC 4.0).

CCopyright: the Author(s), 2021

Licensee PAGEPress, Italy

Biomedical Science and Engineering 2021; 4(s1):189 doi:10.4081/bse.2021.189

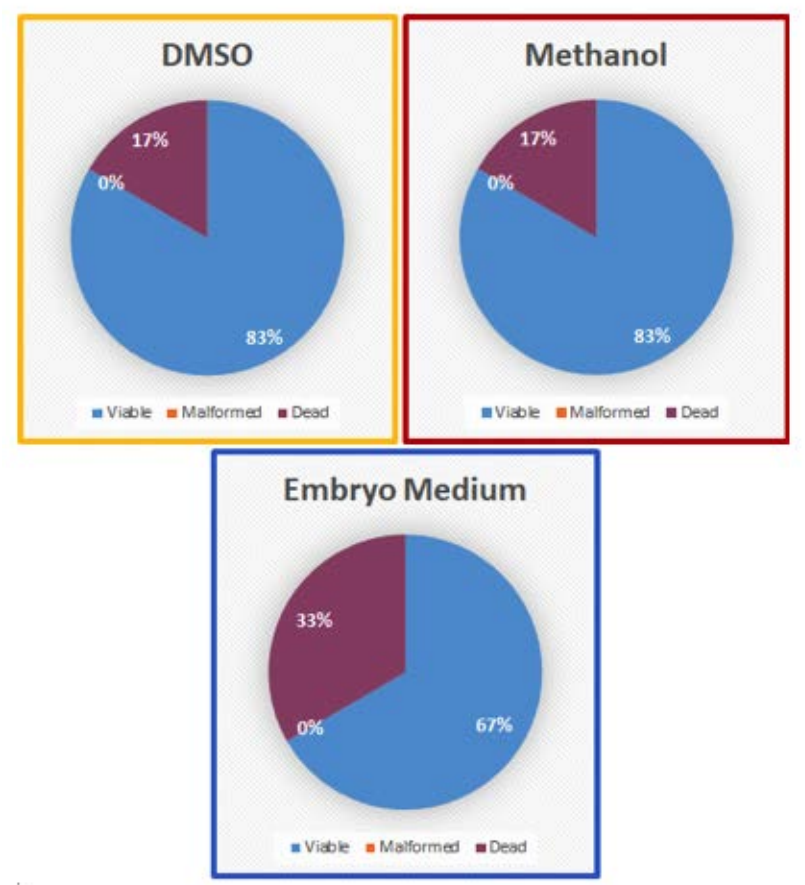

Figure 1. Graphs showing in percentage the mortality and viability values found with incubation in EM and in $0.025 \mathrm{M}$ concentration for both DMSO and $\mathrm{MeOH}$ to which sucrose, at a concentration of $0.2 \mathrm{M}$, has been added. No embryos showing signs attributable to developmental abnormalities or malformations were recorded. 
embryos were transferred in 24 wells plates, each well already containing $2 \mathrm{~mL}$ of solution in the number of one embryo per well. A whole plate was prepared for each experimental condition, so 72 embryos is the total number for the three experimental groups performed: DMSO and $\mathrm{MeOH} 0.025 \mathrm{M}$ added with $0.2 \mathrm{M}$ of sucrose and EM used as negative control.sed as negative control. Thereafter, the plates were incubated for 24 hours at a temperature of $4^{\circ} \mathrm{C}$. The next step was to transfer the embryos to new plates with fresh EM. After that, the plates were brought back at the optimal development temperature of $28.5^{\circ} \mathrm{C}$ for all the duration of the test. At two key moments, $24 \mathrm{hpt}$ and $96 \mathrm{hpt}$, the eggs were visually checked under microscope to assess any sign of malformation or abnormality in the development post chilling.

\section{Results}

The tests performed were many, in order to refine and adjust the parameters of the protocol each time, based on the results generated by the previous ones. Therefore, the results of the last experiment performed were taken into account and here reported. In the last test, thanks to the continuous refinement of the experimental parameters and the constant updating and comparison with the previous outcomes, the data recorded and the relative graphs of the viability and mortality are shown in Figure 1.

These data show how the conditions, starting from the first test setups, have improved with time and practice. In fact, as can be observed from the DMSO and $\mathrm{MeOH}$ graphs in the figure, the embryonic viability was found to be particularly high (although not in all the samples examined). As regards EM on the other hand, the majority of embryos were recorded as viable, but with a consistent percentage of embryonic mortality.

\section{Discussion and Conclusions}

From the data obtained it was crucial, for the refrigerated protocol, not only which one was the best solution to apply and the relative concentration of use, but also the most suitable embryo's developmental stage to be chosen.Moreover, the addition of sucrose to the storage solutions and the relative concentration of use proved to be extremely decisive in the conservation of embryos and in maintaining the post-treatment viability. In conclusion, further and more targeted investigations would be necessary to optimize an effective cooling protocol, as the results obtained so far could pave the way for the improvement and expansion of the application of existing conservation methods.

\section{References}

1. Desai K, Spikings E, Zhang T. Shortterm chilled storage of zebrafish (Danio rerio) embryos in cryoprotectant as an alternative to cryopreservation. Zebrafish 2015;12:111-20.

2. Zhang T, Rawson DM. Studies on chilling sensitivity of zebrafish (Brachydanio rerio) embryos. Cryobiology 1995;32:239-46.

3. do Carmo Faria Paes M, Satiko Okada Nakaghi L. Post-cooling survival, growth and deformity rates in zebrafish embryos (Danio rerio). Zygote 2018;26:76-88. 